\title{
Minimization of fan energy consumption in ventilation and (or) air-conditioning systems as an optimization calculus of variations
}

\author{
Michat $\operatorname{Karpuk}^{1, *}$ \\ ${ }^{1}$ Wroclaw University of Science and Technology, Faculty of Environmental Engineering, \\ Plac Grunwaldzki 13 50-377 Wrocław, Poland
}

\begin{abstract}
The article presents an optimization calculus of variations of fan energy consumption in ventilation and (or) air-conditioning systems. It defines an air flow rate function that depends on the time of operation in the defined room size, starting conditions and the function of hazardous substances emission rate in the room. The differential form of air flow rate dependence on density of hazardous substances allows to establish a connection between air pollution in the room and a fan air flow rate, i.e. fan energy consumption. Creating a fan energy model experiment in the room in different conditions allows to minimize energy consumption to 5-30\% depending on existing conditions.
\end{abstract}

\section{Introduction}

Minimization of energy consumption plays an important role in air ventilation and air-conditioning. This is also related to the minimization of electrical energy consumption required for electrical engine of fans that create air intake to establish the conditions for ventilation and air conditioning of rooms.

Traditionally the rate of consumed air flow $V\left(\mathrm{~m}^{3} / \mathrm{s}\right)$ in ventilation systems is calculated as an equation [1-4]:

$$
V=\frac{K_{s}}{s_{\max }-s_{z}},
$$

where $K_{s}(\mathrm{~kg} / \mathrm{s})$ - capacity of emitted hazardous substances in the room with a size of $P\left(\mathrm{~m}^{3}\right), s_{z}\left(\mathrm{~kg} / \mathrm{m}^{3}\right)$ - density of hazardous substances in the outdoor air intake, $s_{\max }\left(\mathrm{kg} / \mathrm{m}^{3}\right)-$ maximum allowable density of hazardous substances in ventilated air.

If in the equation (1) we exchange $K_{s} \rightarrow Q_{s}(\mathrm{~J} / \mathrm{s})=$ excessive heat in the room, $s \rightarrow I$ (or $s \rightarrow \rho c t)\left(\mathrm{J} / \mathrm{m}^{3}\right)$, where $I$ - density of air enthalpy, $\rho$ - air density, $c$ - specific heat capacity, $t$ - temperature of the ventilated air, an equation of the consumed air volume that depends on indoor and outdoor temperature $[4,5]$ is established.

\footnotetext{
*Corresponding author: michal.karpuk@pwr.edu.pl
} 


$$
V=\frac{Q_{s}}{\rho c\left(t_{\max }-t_{z}\right)},
$$

Equations (1) and (1') have been developed based on the operating conditions of the fan [1], responsible for constant air flow $V\left(\mathrm{~m}^{3} / \mathrm{s}\right)$ and constant emission rate of hazardous substances in the room $K_{s}$, due to which the density of hazardous substances in the ventilated air does not exceed the maximum allowed density $s_{\max }\left(\mathrm{kg} / \mathrm{m}^{3}\right)$.

Equations (1) and (1') present the solution to the difference equation of hazardous substances density balance in the room air [1] for a time period $\tau \in[0, T]$ :

$$
P d s=V s_{z} d \tau+K_{s} d \tau-V s d \tau
$$

Equations (1) and (1') can be used for calculation of ventilation and air-conditioning systems under assumption that a fan pumps the air flow $V\left(\mathrm{~m}^{3} / \mathrm{s}\right)$ on a regular basis. Contemporary electronic control systems can regulate fan operation mode in time. Consequently, to minimize the energy consumption in ventilation and (or) air-conditioning systems it is important to define a task related to the optimization of variable conditions of fan operation (optimal time functions of fan operation) that provides acceptable conditions in the ventilated room depending on the density of emitted hazardous substances.

The purpose of the article is to explore the variable operation modes of fans in the room that consume minimum energy under the given conditions.

\section{Theory}

\subsection{Optimization problem of fan energy consumption minimization}

Electrical capacity of the fan $W$ is proportional to excessive pressure $\Delta p$ of the fan and its air flow consumption rate $V[6]$.

$$
W \square \Delta p \cdot V,
$$

On the other hand, for fans in the operation mode excessive pressure is considered as proportional to the square of air flow consumption rate $\Delta p \sqcap V^{2}$ for simple ventilation systems and to $\Delta p \sqcap V^{n}, n>2$ for the network systems [4].

Therefore, the capacity of the fan equals

$$
W=k V^{1+n},
$$

where $k$ - coefficient of proportionality that depends on the air density and temperature as well as other aspects of the fan.

If we assume that the fan operates for a particular time period $[0, \mathrm{~T}]$, then the energy E of the fan equals

$$
E=f(V, \tau)=\int_{0}^{T} W d \tau=\int_{0}^{T} k V^{1+n} d \tau,
$$

The equation (5) presents the energy consumed for room ventilation. The equation (5) is an objective function in the optimization problem. The problem of ensuring required 
conditions of ventilation $0 \leq s_{z}(\tau) \leq s(\tau) \leq s_{\max }, \tau \in[0, T]$ is presented as a limitation where energy (5) is a function of air flow $V\left(\mathrm{~m}^{3} / \mathrm{s}\right)$ consumed by ventilation system and system operation time $\tau$.

As a result, optimization problem of energy consumption minimization can be presented as the following:

$$
\int_{0}^{T} k V^{1+n}(\tau) d \tau \rightarrow \min ,
$$

with the following limitations

$$
0 \leq s_{z}(\tau) \leq s(\tau) \leq s_{\max }
$$

Minimization problem (6) is a calculus of variations problem [7, 8], the idea of which is to find such a function of consumed airflow $V(\tau)$ with dependence on time $\tau$, that can show the minimum of equation (5) under the condition (7), that states that the density of hazardous substances does not exceed the maximum allowed density in the room with the capacity $P\left(\mathrm{~m}^{3}\right)$.

\subsection{Energy consumption of a fan with a constant airflow}

It can be assumed that the system has a fan that operates in different modes and intakes the air flow that depends on time: $V=V(\tau)$.

Energy $E_{0}$ consumed during fan operation mode that provides a constant air inflow $V$ during the time $\tau$ is defined as a target value. Since $V=$ const, then:

$$
E_{0}=\int_{0}^{T} k V^{1+n} d \tau=k V^{1+n} T
$$

If equation (1) is used in (8), dependence of energy consumption on density of hazardous substances is defined as:

$$
E_{0}=k\left(\frac{K_{s}}{s_{\max }-s_{z}}\right)^{1+n} T
$$

The equation (9) provides several obvious conclusions. First, energy $E_{0}$ consumed for ventilation is proportional to the emission rate of hazardous substances in the room $K_{s}$ to the $(1+n)$ degree. Secondly, energy consumption is growing to the $(1+n)$ degree if the density of hazardous substances $s_{z}$ in the outdoor air intake is too high. Thirdly, energy consumption is growing proportionally to the operating time of the fan $\tau$.

\subsection{Optimization of energy consumption of a fan with a variable airflow}

Possibilities of minimization of energy consumption by means of fan operation mode modification in time are presented in this section. Since in the difference model (2) density of hazardous substances in the room $s=s(V, \tau)$ has a simple dependence on time $\tau$ and air intake $V$, calculus of variations problem (6) can be reduced to one-function problem 
$s=s(\tau)$. For this purpose, variable $V=V(\tau)$ in the equation (6) is excluded after the transformation of the equation (2):

$$
V(\tau)=\frac{K_{s}}{s(\tau)-s_{z}}-\frac{P}{s(\tau)-s_{z}} \frac{d s}{d \tau}=\frac{K_{s}}{s(\tau)-s_{z}}+P \frac{d}{d \tau} \ln \left(s_{z}-s(\tau)\right)
$$

Differential equation (10) allows one to find a function of air intake of the fan $V(\tau)$, if the dependence of density of hazardous substances on time $s=s(\tau)$ is known. If $s=$ const, then the derivative is $\frac{d}{d \tau} \ln \left(s_{z}-s(\tau)\right)=0$, and the equation (10) becomes the equation (1).

The equation (10) can be interpreted as the split of fan air flow $V$ to two components. One part of the air flow is used for the minimization of pollution caused by the internal source of the pollution (first component $\frac{K_{s}}{s(\tau)-s_{z}}-$ inverse dependence from $s$ ). The second part of the air flow (second component $\left.P \frac{d}{d \tau} \ln \left(s_{z}-s(\tau)\right)\right)$ is used for the transformation of the logarithm of pollution. If $s(\tau)$ decreases, then the both components start increasing, which leads to non-linear increase of air flow volume $V$. Similarly, if $s(\tau)$ increases, the first and second components decrease.

The function of minimization of energy consumption $\mathrm{E}$ is:

$$
E(s(\tau))=\int_{0}^{T} k(V(\tau))^{1+n} d \tau=k \int_{0}^{T}\left(\frac{K_{s}}{s(\tau)-S_{z}}-\frac{P}{s(\tau)-s_{z}} \dot{s}(\tau)\right)^{1+n} d \tau \rightarrow \min
$$

where $\dot{s}(\tau)=\frac{d s}{d \tau}$

For air flow intake model experiment we used the data from the article [9]: $V=100000 \mathrm{~m}^{3} / \mathrm{h} \approx 30 \mathrm{~m}^{3} / \mathrm{s}, P=12500 \mathrm{~m}^{3}$. The following data were chosen as well: $K_{s}=K_{s 0}=0.03 \mathrm{~kg} / \mathrm{s}, s_{z}=0.001 \mathrm{~kg} / \mathrm{m}^{3}, s_{\max }=0.002 \mathrm{~kg} / \mathrm{m}^{3}, T=24 \mathrm{~h}, n=3$. For the constant operating mode of a fan, the assumption of the energy consumption is $E_{0}=2.33 \cdot 10^{9} \mathrm{k}(\mathrm{J})$.

Curves $s(\tau)$ and $V(\tau)$ on Fig.1 show possible dependencies (10) and (11). During the time period $[0, \mathrm{~T}]$ density of hazardous substances in the air $s(\tau)$ changes and the limitation (7) is present. At the same time fan operating mode $V(\tau)$ changes with time according to (10). Energy consumption for ventilation is defined by equation (11).

The minimization problem consists in finding among all the potential curves $s(\tau)$ such curves that show the minimum of the function (11), calculated based on the dependence for $V(\tau)$.

To solve the calculus of variations problem (11) one first needs to solve the Euler-Poisson equation [7, 8]

$$
F_{s}-\frac{d}{d \tau} F_{\dot{s}}=0, \quad F(\tau, s, \dot{s})=\left(\frac{K_{s}}{s(\tau)-s_{z}}-\frac{P}{s(\tau)-s_{z}} \dot{s}(\tau)\right)^{1+n}
$$


One can simplify the problem by assuming that $K_{s}=K_{s}(\dot{s})$ exclusively depends on the rate of pollution variation $\dot{S}(\tau)$. In this case the equation (12) is modified to:

$$
F(\tau, s, \dot{s})=\left(s(\tau)-s_{z}\right)^{-1-n}\left(K_{s}-P \dot{s}(\tau)\right)^{1+n}=F_{1}(\tau, s) F_{2}(\tau, \dot{s})
$$

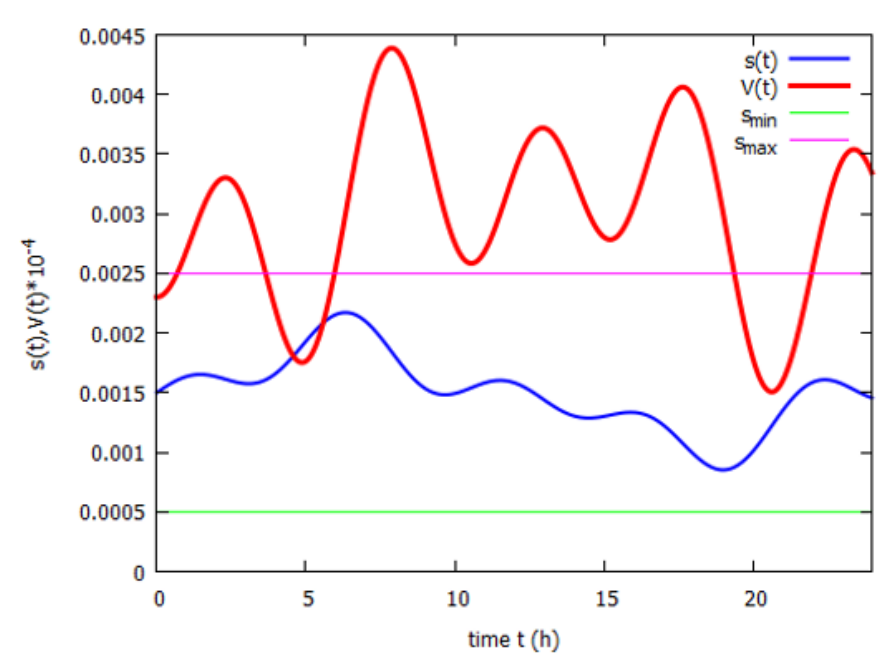

Fig. 1. An example of the dependence of fan air flow intake $V(\tau)$ and density of hazardous substances $s(\tau)$ on time $\tau$.

After the variables in the equation (13) are divided, assuming that $F_{1}(\tau, s)=\left(s(\tau)-s_{z}\right)^{-1-n} \neq 0$, the equation is as follows:

$$
F_{s}-\frac{d}{d \tau} F_{\dot{s}}=F_{1 s}\left(F_{2 \dot{s}}-\frac{d}{d \tau} F_{2 \dot{s}}\right)=0,
$$

Or as follows:

$$
\begin{aligned}
\left(K_{s}-P \dot{s}(\tau)\right)^{1+n}-\frac{d}{d \tau}\left(K_{s}-P \dot{s}(\tau)\right)^{1+n}= & \\
= & \left(K_{s}-P \dot{s}(\tau)\right)^{n}\left(K_{s}-P \dot{s}(\tau)-(1+n)\left(\dot{K}_{s}-P \ddot{s}(\tau)\right)\right)=0
\end{aligned}
$$

The equation (15) allows to find dependence $s(\tau)$, which presents the optimal (minimal) energy consumption. By defining the dependence $s(\tau)$, air flow intake and energy consumption can be calculated based on equations (10) and (11). 


\section{Model experiment of air flow intake and energy consumption}

The equation (15) can be divided into two equations. The first equation $\left(K_{s}-P \dot{s}(\tau)\right)^{n}=0$ shows dependence of hazardous substances $s(\tau)$ on emission rate of hazardous substances in the room $K_{s}$. Integral equation is used to solve this:

$$
s(\tau)=\int_{0}^{\tau} \frac{K_{s}(t)}{P} d t
$$

If equation (16) is used in (10) and (11), trivial solution $V(\tau)=0, E(\tau)=0$ can be found, which means that the need for air ventilation does not exist as the density of hazardous substances in the room for the time period $\mathrm{T}$ does not exceed the allowed density $s_{\max }$.

The second equation is a differential equation of second order:

$$
\ddot{s}(\tau)-\frac{1}{1+n} \dot{s}(\tau)+\frac{K_{s}-(1+n) \dot{K}_{s}}{(1+n) P}=0
$$

\section{1}

In this section the assumption $K_{s}=$ const is examined. $K_{s}=$ const means that the emission rate of hazardous substances is constant and does not depend on time ( $\left.\dot{K}_{s}=0\right)$.

The equation (17) is solved in the following way:

$$
s(\tau)=\frac{K_{s}}{P} \tau-\frac{C_{1}(1+n)}{P} e^{\frac{\tau}{1+n}}+C_{2}
$$

In this case $C_{1}, C_{2}$ are the regular integrations that are present from the starting conditions of $s(\tau)$, for example, in the time period $\tau=0 s(0)=s_{\max }$ or in the time period $\tau=0, s(T)=1.1 s_{z}$ :

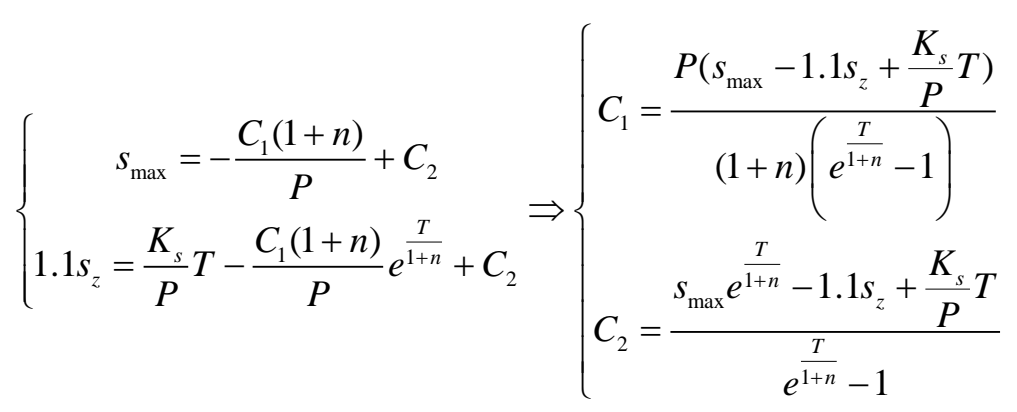

Density of hazardous substances in the room $s(\tau)$ is presented by linear and exponential component. The fan air rate intake is shown as follows:

$$
V(\tau)=\frac{K_{s}-P \frac{d s}{d \tau}}{s(\tau)-s_{z}}=\frac{C_{1} e^{\frac{\tau}{1+n}}}{\frac{K_{s}}{P} \tau-\frac{C_{1}(1+n)}{P} e^{\frac{\tau}{1+n}}+C_{2}-s_{z}}
$$


Integration of the equation (8) upon the dependence (20) defines the energy consumed by the fan.

Numerical model experiment for the abovementioned data and starting conditions shows the following result: $E_{l}=1.647 \cdot 10^{9} \mathrm{k}(\mathrm{J})$, where $E_{l} / E_{0}=0.707=70.7 \%$. Consequently, the use of optimal function $V(\tau)$ allows to decrease fan energy consumption by approximately $30 \%$. Fig. 2 shows dependencies $V(\tau)$ and $s(\tau)$. Since the operation mode $s(0)=s_{\max }, s(T)=1.1 s_{z}$ has been chosen, the $60 \%$ of fan operation is directed to keep $s=s(0)$, and for the $40 \%$ of the remaining time the fan increases its capacity to decrease $s$ to the level of $s(T)=1.1 s_{z}$.

Operating mode for different $s(0), s(T)$ is calculated in a similar way.

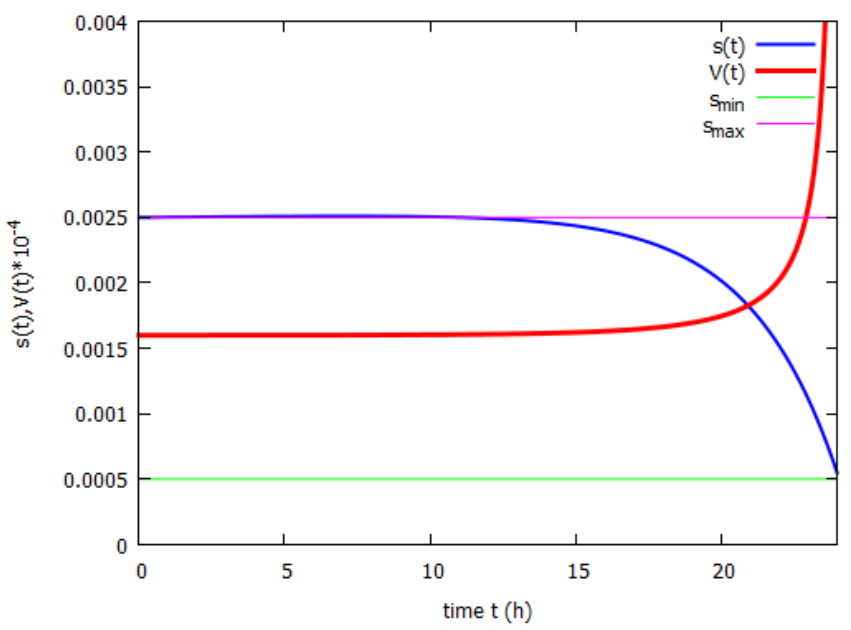

Fig. 2. Dependence of fan air flow intake $V(\tau)$ and density of hazardous substances $s(\tau)$ on time $\tau$ for the assumption $K_{s}=$ const, $s(0)=s_{\max }, s(\mathrm{~T})=1.1 s_{z}$.

\section{2}

In this section assumption $K_{s}=K_{s 0}\left(1+\sin \left(\pi \frac{\tau}{T}\right)\right)$ is examined. This assumption shows that the emission rate of hazardous substances in the time period $T$ changes harmonically and reaches the maximum of $2 K_{s 0}$ at the time period $T / 2$.

The solution to the equation (17) is

$$
\left.s(\tau)=\frac{K_{s 0}}{P}\left(\tau-\frac{T}{\pi} \cos \left(\pi \frac{\tau}{T}\right)\right)\right)-\frac{C_{1}(1+n)}{P} e^{\frac{\tau}{1+n}}+C_{2}
$$

Based on this equation, optimal function $V(\tau)$ is calculated from equation (10). Correlation between $E_{l} / E_{0}$ equals $94.2 \%$.

\section{Conclusions}

The problem of the fan energy consumption minimalization has been approached as a calculus of variations problem. This approach has allowed to identify air flow rate function $V(\tau)$ depending on the time and defined room size, initial conditions and the function of hazardous substances emission rate in the room $K_{s}(\tau)$. 
The differential form (10) of air flow rate dependence $V(\tau)$ on hazardous substances density $s(\tau)$ has allowed to establish a connection between air pollution in the room and a fan air flow rate and, consequently, a fan energy consumption.

The model experiment of a fan energy in the room in different operation modes has shown the possibility to minimize energy consumption by $5-30 \%$ depending on operating conditions.

\section{References}

1. V. V. Baturin, Osnowy promyschlennoj ventilacii (Profizdat, Moskva, 1990)

2. S. Przydróżny, Wentylacja (Wydawnictwo Politechniki Wrocławskiej, 1991)

3. H. Goodfellow, E. Tahti, Industrial Ventilation (Academic Press, 2001)

4. K. Shan, K. Wang, Handbook of air conditioning and refrigeration $\left(2^{\text {nd }}\right.$ ed. McGraw-Hill, 2001)

5. A. Pełech, Wentylacja i Klimatyzacja. Podstawy (Oficyna Wydawnicza Politechniki Wrocławskiej, 2009)

6. Laboratory method of testing fans for aerodynamic performance rating. Air Movement and Control Association International, Inc. and American Society of Heating, Refrigerating and Air Conditioning Engineers, Inc. ANSI/AMCA STANDARD 210 ANSI/ASHRAE STANDARD 51 (1999)

7. G. A. Bliss, Lectures on the Calculus of Variations (University of Chicago Press, Chicago, 1946)

8. I. M. Gelfand, S. V. Fomin, Calculus of Variations (Prentice Hall, 1963)

9. M. Karpuk, A. Pełech, E. Przydróżny, J. Walaszczyk, S. Szczęśniak, ASEE17, E3S Web of Conferences 22, 00078 (2017) 